Kragujevac Journal of Mathematics

Volume 39(2) (2015), Pages 217-230.

\title{
COMPACTNESS OF MATRIX OPERATORS ON SOME SEQUENCE SPACES DERIVED BY FIBONACCI NUMBERS
}

\author{
E. E. KARA ${ }^{1}$, M. BAŞARIR ${ }^{2}$, AND M. MURSALEEN ${ }^{3}$
}

\begin{abstract}
In this paper, we apply the Hausdorff measure of noncompactness to obtain the necessary and sufficient conditions for certain matrix operators on the Fibonacci difference sequence spaces $\ell_{p}(\widehat{F})$ and $\ell_{\infty}(\widehat{F})$ to be compact, where $1 \leq p<\infty$
\end{abstract}

\section{Introduction AND PRELIMINARIES}

Let $\mathbb{N}=\{0,1,2, \ldots\}$ and $\mathbb{R}$ be the set of all real numbers. We shall write $\lim _{k}$, $\sup _{k}, \inf _{k}$ and $\sum_{k}$ instead of $\lim _{k \rightarrow \infty}, \sup _{k \in \mathbb{N}}, \inf _{k \in \mathbb{N}}$ and $\sum_{k=0}^{\infty}$, respectively. Let $\omega$ be the vector space of all real sequences $x=\left(x_{k}\right)_{k \in \mathbb{N}}$. By the term sequence space, we shall mean any linear subspace of $\omega$. Let $\varphi, \ell_{\infty}, c$ and $c_{0}$ denote the sets of all finite, bounded, convergent and null sequences, respectively. We write $\ell_{p}=\left\{x \in \omega: \sum_{k}\left|x_{k}\right|^{p}<\infty\right\}$ for $1 \leq p<\infty$. Also, we shall use the conventions that $e=(1,1, \ldots)$ and $e^{(n)}$ is the sequence whose only non-zero term is 1 in the $n^{\text {th }}$ place for each $n \in \mathbb{N}$. For any sequence $x=\left(x_{k}\right)$, let $x^{[n]}=\sum_{k=0}^{n} x_{k} e^{(k)}$ be its $n$-section. Morever, we write $b s$ and $c s$ for the sets of series with bounded and convergent partial sums, respectively.

The Fibonacci numbers are the sequence of numbers $\left\{f_{n}\right\}_{n=0}^{\infty}$ defined by the linear recurrence equations

$$
f_{0}=f_{1}=1 \text { and } f_{n}=f_{n-1}+f_{n-2} ; \quad n \geq 2 .
$$

Key words and phrases. Sequence spaces, Fibonacci numbers, compact operators, Hausdorff measure of noncompactness.

2010 Mathematics Subject Classification. Primary: 46A45, 11B39, 46B50.

Received: March 6, 2015.

Accepted: August 29, 2015. 
Fibonacci numbers have many interesting properties and applications in arts, sciences and architecture. For example, the ratio sequences of Fibonacci numbers converge to the golden ratio which is important in sciences and arts. Also, some basic properties of Fibonacci numbers can be found in [19].

A $B-$ space is a complete normed space. A topological sequence space in which all coordinate functionals $\pi_{k}, \pi_{k}(x)=x_{k}$, are continuous is called a $K-$ space. A $B K-$ space is defined as a $K$ - space which is also a $B$ - space, that is, a $B K$ - space is a Banach space with continuous coordinates. A $B K$ - space $X \supset \varphi$ is said to have $A K$ if every sequence $x=\left(x_{k}\right) \in X$ has a unuqiue representation $x=\sum_{k} x_{k} e^{(k)}$. For example, the space $\ell_{p}(1 \leq p<\infty)$ is $B K-$ space with $\|x\|_{p}=\left(\sum_{k}\left|x_{k}\right|^{p}\right)^{1 / p}$ and $c_{0}$, $c$ and $\ell_{\infty}$ are $B K-$ spaces with $\|x\|_{\infty}=\sup _{k}\left|x_{k}\right|$. Further, the $B K-$ spaces $c_{0}$ and $\ell_{p}$ have $A K$, where $1 \leq p<\infty([22,4])$.

A sequence $\left(b_{n}\right)$ in a normed space $X$ is called a Schauder basis for $X$ if for every $x \in X$ there is a unique sequence $\left(\alpha_{n}\right)$ of scalars such that $x=\sum_{n} \alpha_{n} b_{n}$, i.e., $\lim _{m}\left\|x-\sum_{n=0}^{m} \alpha_{n} b_{n}\right\|=0$.

The $\beta$-dual of a sequence space $X$ is defined by

$$
X^{\beta}=\left\{a=\left(a_{k}\right) \in \omega: a x=\left(a_{k} x_{k}\right) \in c s \text { for all } x=\left(x_{k}\right) \in X\right\} .
$$

Let $A=\left(a_{n k}\right)_{n, k=0}^{\infty}$ be an infinite matrix of real numbers $a_{n k}$, where $n, k \in \mathbb{N}$. We write $A_{n}$ for the sequence in the $n^{\text {th }}$ row of $A$, that is $A_{n}=\left(a_{n k}\right)_{k=0}^{\infty}$ for every $n \in \mathbb{N}$. In addition, if $x=\left(x_{k}\right)_{k=0}^{\infty} \in \omega$ then we define the $A$-transform of $x$ as the sequence $A x=\left\{A_{n}(x)\right\}_{n=0}^{\infty}$, where

$$
A_{n}(x)=\sum_{k=0}^{\infty} a_{n k} x_{k} ; \quad n \in \mathbb{N}
$$

provided the series on the right side converges for each $n \in \mathbb{N}$.

For arbitrary subsets $X$ and $Y$ of $\omega$, we write $(X, Y)$ for the class of all infinite matrices that map $X$ into $Y$. Thus, $A \in(X, Y)$ if and only if $A_{n} \in X^{\beta}$ for all $n \in \mathbb{N}$ and $A x \in Y$ for all $x \in X$.

The matrix domain $X_{A}$ of an infinite matrix $A$ in a sequence space $X$ is defined by

$$
X_{A}=\left\{x=\left(x_{k}\right) \in \omega: A x \in X\right\}
$$

which is a sequence space ([4]).

Let $\Delta$ denote the matrix $\Delta=\left(\Delta_{n k}\right)$ defined by

$$
\Delta_{n k}= \begin{cases}(-1)^{n-k}, & (n-1 \leq k \leq n), \\ 0, & (0 \leq k<n-1) \text { or }(k>n),\end{cases}
$$

or

$$
\Delta_{n k}= \begin{cases}(-1)^{n-k}, & (n \leq k \leq n+1), \\ 0, & (0 \leq k<n) \text { or }(k>n+1) .\end{cases}
$$

In the literature, the matrix domain $\lambda_{\Delta}$ is called the difference sequence space whenever $\lambda$ is a normed or paranormed sequence space. The idea of difference sequence 
space was introduced by Kızmaz [17]. In 1981, Kızmaz [17] defined the sequence spaces

$$
X(\Delta)=\left\{x=\left(x_{k}\right) \in \omega:\left(x_{k}-x_{k+1}\right) \in X\right\}
$$

for $X=\ell_{\infty}, c$ and $c_{0}$. The difference space $b v_{p}$, consisting of all sequnces $\left(x_{k}\right)$ such that $\left(x_{k}-x_{k-1}\right)$ is in the sequence space $\ell_{p}$, was studied in the case $0<p<1$ by Altay and Başar [2] and in the case $1 \leq p \leq \infty$ by Başar and Altay [5] and Çolak et al. [12]. The paranormed difference sequence space

$$
\Delta \lambda(p)=\left\{x=\left(x_{k}\right) \in \omega:\left(x_{k}-x_{k+1}\right) \in \lambda(p)\right\}
$$

was examined by Ahmad and Mursaleen [1] and Malkowsky [23], where $\lambda(p)$ is any of the paranormed spaces $\ell_{\infty}(p), c(p)$ and $c_{0}(p)$ defined by Simons [37] and Maddox [20].

Recently, Başar et al. [3] have defined the sequence spaces $b v(u, p)$ and $b v_{\infty}(u, p)$ by

$$
b v(u, p)=\left\{x=\left(x_{k}\right) \in \omega: \sum_{k}\left|u_{k}\left(x_{k}-x_{k-1}\right)\right|^{p_{k}}<\infty\right\}
$$

and

$$
b v_{\infty}(u, p)=\left\{x=\left(x_{k}\right) \in \omega: \sup _{k \in \mathbb{N}}\left|u_{k}\left(x_{k}-x_{k-1}\right)\right|^{p_{k}}<\infty\right\},
$$

where $u=\left(u_{k}\right)$ is an arbitrary fixed sequence and $0<p_{k} \leq H<\infty$ for all $k \in \mathbb{N}$. Also in $[11,13,14,18,24,25,27,28,36,38]$, authors studied some difference sequence spaces.

Let $S_{X}$ denote the unit sphere in a normed linear space $X$. If $X \supset \varphi$ is a $B K$ space and $a=\left(a_{k}\right) \in \omega$, then we write

$$
\|a\|_{X}^{*}=\sup _{x \in S_{X}}\left|\sum_{k} a_{k} x_{k}\right|
$$

provided the expression on the right side is defined and finite which is the case whenever $a \in X^{\beta}$.

The following results are very important in our study.

Lemma 1.1. [22, Theorem 1.29] Let $1<p<\infty$ and $q=p /(p-1)$. Then, we have $\ell_{\infty}^{\beta}=\ell_{1}, \ell_{1}^{\beta}=\ell_{\infty}$ and $\ell_{p}^{\beta}=\ell_{q}$. Furthermore, let $X$ denote any of the spaces $\ell_{\infty}, \ell_{1}$ or $\ell_{p}$. Then, we have $\|a\|_{X}^{*}=\|a\|_{X^{\beta}}$ for all $a \in X^{\beta}$, where $\|\cdot\|_{X^{\beta}}$ is the natural norm on the dual space $X^{\beta}$.

Lemma 1.2. [22, Theorem 1.23 (a)] Let $X$ and $Y$ be BK-spaces. Then we have $(X, Y) \subset B(X, Y)$, that is, every matrix $A \in(X, Y)$ defines a linear operator $L_{A} \in$ $B(X, Y)$ by $L_{A}(x)=A x$ for all $x \in X$, where $B(X, Y)$ denotes the set all bounded (continuous) linear operators $L: X \rightarrow Y$.

Lemma 1.3. [22, Lemma 2.2] Let $X \supset \phi$ be BK-space and $Y$ be any of the spaces $c_{0}, c$ or $\ell_{\infty}$. If $A \in(X, Y)$, then 


$$
\left\|L_{A}\right\|=\|A\|_{\left(X, \ell_{\infty}\right)}=\sup _{n}\left\|A_{n}\right\|_{X}^{*}<\infty .
$$

By $M_{X}$, we denote the collection of all bounded subsets of a metric space $(X, d)$. If $Q \in M_{X}$, then the Hausdorff measure of noncompactness of the set $Q$, denoted by $\chi(Q)$, is defined by

$$
\chi(Q)=\inf \left\{\varepsilon>0: Q \subset \bigcup_{i=1}^{n} B\left(x_{i}, r_{i}\right), x_{i} \in X, r_{i}<\varepsilon,(i=1,2, \ldots, n), n \in \mathbb{N}-\{0\}\right\} .
$$

The function $\chi: M_{X} \rightarrow[0, \infty)$ is called the Hausdorff measure of noncompactness.

The basic properties of the Hausdorff measure of noncompactness can be found in [22].

The following result gives an estimate for the Hausdorff measure of noncompactness in the $B K$ space $\ell_{p}$ for $1 \leq p<\infty$.

Lemma 1.4. [35, Theorem 2.8] Let $1 \leq p<\infty$ and $Q \in M_{\ell_{p}}$. If $P_{m}: \ell_{p} \rightarrow \ell_{p}(m \in \mathbb{N})$ is the operator defined by $P_{m}(x)=\left(x_{0}, x_{1}, \ldots, x_{m}, 0,0, \ldots\right)$ for all $x=\left(x_{k}\right) \in \ell_{p}$, then we have

$$
\chi(Q)=\lim _{m \rightarrow \infty}\left(\sup _{x \in Q}\left\|\left(I-P_{m}\right)(x)\right\|_{\ell_{p}}\right),
$$

where $I$ is the identity operator on $\ell_{p}$.

Let $X$ and $Y$ be Banach spaces. Then, a linear operator $L: X \rightarrow Y$ is said to be compact if the domain of $L$ is all of $X$ and $L(Q)$ is a totally bounded subset of $Y$ for every $Q \in M_{X}$. Equivalently, we say that $L$ is compact if its domain is all of $X$ and for every bounded sequence $\left(x_{n}\right)$ in $X$, the sequence $\left(L\left(x_{n}\right)\right)$ has a convergent subsequence in $Y$.

The idea of compact operators between Banach spaces is closely related to the Hausdorff measure of noncompactness, and it can be given as follows.

Let $X$ and $Y$ be Banach spaces and $L \in B(X, Y)$. Then, the Hausdorff measure of noncompactness of $L$, is denoted by $\|L\|_{\chi}$, can be given by

$$
\|L\|_{\chi}=\chi\left(L\left(S_{X}\right)\right)
$$

and we have

$$
L \text { is compact if and only if }\|L\|_{\chi}=0 .
$$

The Hausdorff measure of noncompactness has various applications in the theory of sequence spaces, one of them is to obtain necessary and sufficient conditions for matrix operators between $B K$ spaces to be compact. Recently, several authors have studied compact operators on the sequence spaces and given very important results related to the Hausdorff measure of noncompactness of a linear operator. For example [6-10,16,21,26,29-34].

In this paper, we derive some identities for the Hausdorff measure of noncompactness on the Fibonacci difference sequence spaces $\ell_{p}(\widehat{F})$ and $\ell_{\infty}(\widehat{F})$ defined by Kara [15]. 
We also apply the Hausdorff measure of noncompactness to obtain the necessary and sufficient conditions for such operators to be compact.

\section{The Fibonacci Difference Sequence Spaces $\ell_{p}(\widehat{F})$ And $\ell_{\infty}(\widehat{F})$}

Throughout, let $1 \leq p \leq \infty$ and $q$ denote the conjugate of $p$, that is, $q=p /(p-1)$ for $1<p<\infty, q=\infty$ for $p=1$ or $q=1$ for $p=\infty$.

Recently, Kara[15] has defined the Fibonacci difference sequence spaces $\ell_{p}(\widehat{F})$ and $\ell_{\infty}(\widehat{F})$ by

$$
\ell_{p}(\widehat{F})=\left\{x=\left(x_{n}\right) \in \omega: \sum_{n}\left|\frac{f_{n}}{f_{n+1}} x_{n}-\frac{f_{n+1}}{f_{n}} x_{n-1}\right|^{p}<\infty\right\} ; \quad 1 \leq p<\infty
$$

and

$$
\ell_{\infty}(\widehat{F})=\left\{x=\left(x_{n}\right) \in \omega: \sup _{n \in \mathbb{N}}\left|\frac{f_{n}}{f_{n+1}} x_{n}-\frac{f_{n+1}}{f_{n}} x_{n-1}\right|<\infty\right\} .
$$

With the notation of $(1.2)$, the sequence spaces $\ell_{p}(\widehat{F})$ and $\ell_{\infty}(\widehat{F})$ may be redefined by

$$
\ell_{p}(\widehat{F})=\left(\ell_{p}\right)_{\widehat{F}}(1 \leq p<\infty) \text { and } \ell_{\infty}(\widehat{F})=\left(\ell_{\infty}\right)_{\widehat{F}},
$$

where the matrix $\widehat{F}=\left(\widehat{f}_{n k}\right)$ is defined by

$$
\widehat{f}_{n k}=\left\{\begin{array}{ll}
-\frac{f_{n+1}}{f_{n}}, & k=n-1, \\
\frac{f_{n}}{f_{n+1}}, & k=n, \\
0, & 0 \leq k<n-1 \text { or } k>n,
\end{array} \quad n, k \in \mathbb{N} .\right.
$$

Further, it is clear that the spaces $\ell_{p}(\widehat{F})$ and $\ell_{\infty}(\widehat{F})$ are $B K$ spaces with the norms given by

$$
\|x\|_{\ell_{p}(\widehat{F})}=\left(\sum_{n}\left|y_{n}\right|^{p}\right)^{1 / p} ; 1 \leq p<\infty \text { and }\|x\|_{\ell_{\infty}(\widehat{F})}=\sup _{n}\left|y_{n}\right|,
$$

where the sequence $y=\left(y_{n}\right)=\left(\widehat{F}_{n}(x)\right)$ is the $\widehat{F}$-transform of a sequence $x=\left(x_{n}\right)$, i.e.,

$$
y_{n}=\widehat{F}_{n}(x)=\left\{\begin{array}{ll}
\frac{f_{0}}{f_{1}} x_{0}=x_{0}, & n=0, \\
\frac{f_{n}}{f_{n+1}} x_{n}-\frac{f_{n+1}}{f_{n}} x_{n-1}, & n \geq 1,
\end{array} \quad n \in \mathbb{N} .\right.
$$

Moreover, it is obvious by (2.2) that $\widehat{F}$ is a triangle. Thus, it has a unique inverse $\widehat{F}^{-1}=\left(\widehat{f}_{n k}^{-1}\right)$ which is also a triangle and the elements of $\widehat{F}^{-1}$ are given by

$$
\widehat{f}_{n k}^{-1}= \begin{cases}\frac{f_{n+1}^{2}}{f_{k} f_{k+1}}, & 0 \leq k \leq n \\ 0, & k>n\end{cases}
$$


for all $n, k \in \mathbb{N}$. Therefore, we have by (2.4) that

$$
x_{n}=\sum_{k=0}^{n} \frac{f_{n+1}^{2}}{f_{k} f_{k+1}} y_{k} ; \quad n \in \mathbb{N} .
$$

In [15], the $\beta$-duals of the sequence spaces $\ell_{p}(\widehat{F})(1 \leq p<\infty)$ and $\ell_{\infty}(\widehat{F})$ have been determined and some related matrix classes characterized. Now, by taking into account that the inverse of $\widehat{F}$ is given by (2.5), we have the following lemma which is immediate by [15, Theorem 4.6].

Lemma 2.1. Let $1 \leq p \leq \infty$. If $a=\left(a_{k}\right) \in\left\{\ell_{p}(\widehat{F})\right\}^{\beta}$, then $\bar{a}=\left(\tilde{a}_{k}\right) \in \ell_{q}$ and we have

$$
\sum_{k} a_{k} x_{k}=\sum_{k} \tilde{a}_{k} y_{k}
$$

for all $x=\left(x_{k}\right) \in \ell_{p}(\widehat{F})$ with $y=\widehat{F} x$, where

$$
\tilde{a}_{k}=\sum_{j=k}^{\infty} \frac{f_{j+1}^{2}}{f_{k} f_{k+1}} a_{j} ; \quad k \in \mathbb{N} .
$$

Now, we prove the following results which will be needed in the sequel.

Lemma 2.2. Let $1<p<\infty, q=p /(p-1)$ and $\bar{a}=\left(\tilde{a}_{k}\right)$ be the sequence defined by (2.8). Then, we have

(a) If $a=\left(a_{k}\right) \in\left\{\ell_{\infty}(\widehat{F})\right\}^{\beta}$, then $\|a\|_{\ell_{\infty}(\widehat{F})}^{*}=\sum_{k}\left|\tilde{a}_{k}\right|<\infty$.

(b) If $a=\left(a_{k}\right) \in\left\{\ell_{1}(\widehat{F})\right\}^{\beta}$, then $\|a\|_{\ell_{1}(\widehat{F})}^{*}=\sup _{k}\left|\tilde{a}_{k}\right|<\infty$.

(c) If $a=\left(a_{k}\right) \in\left\{\ell_{p}(\widehat{F})\right\}^{\beta}$, then $\|a\|_{\ell_{p}(\widehat{F})}^{*}=\left(\sum_{k}\left|\tilde{a}_{k}\right|^{q}\right)^{1 / q}<\infty$.

Proof. (a) Let $a=\left(a_{k}\right) \in\left\{\ell_{\infty}(\widehat{F})\right\}^{\beta}$. Then, it follows by Lemma 2.1 that $\bar{a}=\left(\tilde{a}_{k}\right) \in \ell_{1}$ and the equality $(2.7)$ holds for all sequences $x=\left(x_{k}\right) \in \ell_{\infty}(\widehat{F})$ and $y=\left(y_{k}\right) \in \ell_{\infty}$ which are connected by the relation (2.4). Further, we have by $(2.3)$ that $x \in S_{\ell_{\infty}(\widehat{F})}$ if and only if $y \in S_{\ell_{\infty}}$. Therefore, we derive from (1.3) and (2.7) that

$$
\|a\|_{\ell_{\infty}(\widehat{F})}^{*}=\sup _{x \in S_{\ell_{\infty}(\widehat{F})}}\left|\sum_{k} a_{k} x_{k}\right|=\sup _{y \in S_{\ell_{\infty}}}\left|\sum_{k} \tilde{a}_{k} y_{k}\right|=\|\bar{a}\|_{\ell_{\infty}}^{*} .
$$

Hence, by using Lemma 1.1, we have that

$$
\|a\|_{\ell_{\infty}(\widehat{F})}^{*}=\|\bar{a}\|_{\ell_{\infty}}^{*}=\|\bar{a}\|_{\ell_{1}} .
$$

This completes the proof of part (a).

Since parts (b) and (c) can also be proved by analogy with part (a), we leave the detailed proof to the reader. 
Throughout this paper, if $A=\left(a_{n k}\right)$ is an infinite matrix, we define the associated matrix defined $\tilde{A}=\left(\tilde{a}_{n k}\right)$ by

$$
\tilde{a}_{n k}=\sum_{j=k}^{\infty} \frac{f_{j+1}^{2}}{f_{k} f_{k+1}} a_{n j} ; \quad n, k \in \mathbb{N}
$$

provided the series on the right side converges for all $n, k \in \mathbb{N}$ which is the case whenever $A_{n} \in\left\{\ell_{p}(\widehat{F})\right\}^{\beta}$ for all $n \in \mathbb{N}$, where $1 \leq p \leq \infty$. Then, we have:

Lemma 2.3. Let $1 \leq p \leq \infty, X$ be a sequence space and $A=\left(a_{n k}\right)$ be an infinite matrix. If $A \in\left(\ell_{p}(\widehat{F}), X\right)$, then $\tilde{A} \in\left(\ell_{p}, X\right)$ such that $A x=\tilde{A} y$ for all $x \in \ell_{p}(\widehat{F})$ with $y=\widehat{F} x$, where $\tilde{A}=\left(\tilde{a}_{n k}\right)$ is the associated matrix defined by $(2.9)$.

Proof. Suppose that $A \in\left(\ell_{p}(\widehat{F}), X\right)$ and let $x \in \ell_{p}(\widehat{F})$. Then $A_{n} \in\left\{\ell_{p}(\widehat{F})\right\}^{\beta}$ for all $n \in \mathbb{N}$. Thus, it follows by Lemma 2.1 that $\tilde{A}_{n} \in \ell_{q}$ for all $n \in \mathbb{N}$ and the equality $A x=\tilde{A} y$ holds which yields that $\tilde{A} y \in X$, where $y=\widehat{F} x$. Since every $y \in \ell_{p}$ is the assocaited sequence of some $x \in \ell_{p}(\widehat{F})$, we obtain that $\tilde{A} \in\left(\ell_{p}, X\right)$. This concludes the proof.

Lemma 2.4. Let $1 \leq p<\infty$. If $A \in\left(\ell_{1}(\widehat{F}), \ell_{p}\right)$, then

$$
\left\|L_{A}\right\|=\|A\|_{\left(\ell_{1}(\widehat{F}), \ell_{p}\right)}=\sup _{k}\left(\sum_{n}\left|\tilde{a}_{n k}\right|^{p}\right)^{1 / p}<\infty .
$$

Proof. The proof is elementary and left to the reader.

\section{Compact Operators on The Spaces $\ell_{p}(\widehat{F})$ AND $\ell_{\infty}(\widehat{F})$}

In this section, we give some classes of compact operators on the spaces $\ell_{p}(\widehat{F})$ and $\ell_{\infty}(\widehat{F})$, where $1 \leq p<\infty$.

The following lemma gives necessary and sufficient conditions for a matrix transformation from a $B K$ space $X$ to $c_{0}, c$ and $\ell_{\infty}$ to be compact (the only sufficient condition for $\left.\ell_{\infty}\right)$.

Lemma 3.1. [29, Theorem 3.7] Let $X \supset \varphi$ be a BK space. Then, we have

(a) If $A \in\left(X, \ell_{\infty}\right)$, then $0 \leq\left\|L_{A}\right\|_{\chi} \leq \limsup _{n}\left\|A_{n}\right\|_{X}^{*}$.

(b) If $A \in\left(X, c_{0}\right)$, then $\left\|L_{A}\right\|_{\chi}=\limsup \left\|A_{n}\right\|_{X}^{*}$.

(c) If $X$ has $A K$ or $X=\ell_{\infty}$ and $A \stackrel{n}{\in}(X, c)$, then

$$
\frac{1}{2} \cdot \limsup _{n}\left\|A_{n}-\alpha\right\|_{X}^{*} \leq\left\|L_{A}\right\|_{\chi} \leq \limsup _{n}\left\|A_{n}-\alpha\right\|_{X}^{*},
$$

where $\alpha=\left(\alpha_{k}\right)$ with $\alpha_{k}=\lim _{n} a_{n k}$ for all $k \in \mathbb{N}$.

Now, let $A=\left(a_{n k}\right)$ be an infinite matrix and $\tilde{A}=\left(\tilde{a}_{n k}\right)$ the associated matrix defined by (2.9). Then, by combining Lemmas 2.2, 2.3 and 3.1, we have the following result. 
Theorem 3.1. Let $1<p<\infty$ and $q=p /(p-1)$. Then we have:

(a) if $A \in\left(\ell_{p}(\widehat{F}), \ell_{\infty}\right)$, then

$$
0 \leq\left\|L_{A}\right\|_{\chi} \leq \limsup _{n}\left(\sum_{k}\left|\tilde{a}_{n k}\right|^{q}\right)^{1 / q}
$$

and

$$
L_{A} \text { is compact if } \lim _{n}\left(\sum_{k}\left|\tilde{a}_{n k}\right|^{q}\right)^{1 / q}=0 \text {; }
$$

(b) if $A \in\left(\ell_{p}(\widehat{F}), c_{0}\right)$, then

$$
\left\|L_{A}\right\|_{\chi}=\limsup _{n}\left(\sum_{k}\left|\tilde{a}_{n k}\right|^{q}\right)^{1 / q}
$$

and

$$
L_{A} \text { is compact if and only if } \lim _{n}\left(\sum_{k}\left|\tilde{a}_{n k}\right|^{q}\right)^{1 / q}=0 \text {; }
$$

(c) if $A \in\left(\ell_{p}(\widehat{F}), c\right)$, then

$$
\frac{1}{2} \cdot \limsup _{n}\left(\sum_{k}\left|\tilde{a}_{n k}-\tilde{\alpha}_{k}\right|^{q}\right)^{1 / q} \leq\left\|L_{A}\right\|_{\chi} \leq \limsup _{n}\left(\sum_{k}\left|\tilde{a}_{n k}-\tilde{\alpha}_{k}\right|^{q}\right)^{1 / q}
$$

and

$$
L_{A} \text { is compact if and only if } \lim _{n}\left(\sum_{k}\left|\tilde{a}_{n k}-\tilde{\alpha}_{k}\right|^{q}\right)^{1 / q}=0 \text {, }
$$

where $\tilde{\alpha}=\left(\tilde{\alpha}_{k}\right)$ with $\tilde{\alpha}_{k}=\lim _{n} \tilde{a}_{n k}$ for all $k \in \mathbb{N}$.

Proof. It is obvious that (3.2), (3.4) and (3.6) are respectively obtained from (3.1), (3.3) and (3.5) by using (1.5). Thus, we have to prove (3.1), (3.3) and (3.5).

Let $A \in\left(\ell_{p}(\widehat{F}), \ell_{\infty}\right)$ or $A \in\left(\ell_{p}(\widehat{F}), c_{0}\right)$. Since $A_{n} \in\left\{\ell_{p}(\widehat{F})\right\}^{\beta}$ for all $n \in \mathbb{N}$, we have from Part (c) of Lemma 2.2 that

$$
\left\|A_{n}\right\|_{\ell_{p}(\widehat{F})}^{*}=\left\|\tilde{A}_{n}\right\|_{\ell_{p}}^{*}=\left(\sum_{k}\left|\tilde{a}_{n k}\right|^{q}\right)^{1 / q}
$$

for all $n \in \mathbb{N}$. Hence, by using the equality (3.7), we get (3.1) and (3.3) from parts (a) and (b) of Lemma 3.1, respectively.

To prove (3.5), we have $A \in\left(\ell_{p}(\widehat{F}), c\right)$ and hence $\tilde{A} \in\left(\ell_{p}, c\right)$ by Lemma 2.3. Therefore, it follows by part (c) of Lemma 3.1 with Lemma 1.1 that

$$
\frac{1}{2} \cdot \limsup _{n}\left\|\tilde{A}_{n}-\tilde{\alpha}\right\|_{\ell_{q}} \leq\left\|L_{\tilde{A}}\right\|_{\chi} \leq \limsup _{n}\left\|\tilde{A}_{n}-\tilde{\alpha}\right\|_{\ell_{q}},
$$


where $\tilde{\alpha}=\left(\tilde{\alpha}_{k}\right)$ with $\tilde{\alpha}_{k}=\lim _{n} \tilde{a}_{n k}$ for all $k \in \mathbb{N}$.

Now, let us write $S=S_{\ell_{p}(\widehat{F})}$ and $\bar{S}=S_{\ell_{p}}$, for short. Then, we obtain by (1.4) and Lemma 1.2 that

$$
\left\|L_{A}\right\|_{\chi}=\chi\left(L_{A}(S)\right)=\chi(A S)
$$

and

$$
\left\|L_{\tilde{A}}\right\|_{\chi}=\chi\left(L_{\tilde{A}}(\bar{S})\right)=\chi(\tilde{A} \bar{S}) .
$$

Further, we have by (2.3) that $x \in S$ if and only if $y \in \bar{S}$ and since $A x=\tilde{A} y$ by Lemma 2.3, we deduce that $A S=\tilde{A} \bar{S}$. This leads us with (3.9) and (3.10) to the consequence that $\left\|L_{A}\right\|_{\chi}=\left\|L_{\tilde{A}}\right\|_{\chi}$. Hence, we get (3.5) from (3.8). This completes the proof.

The conclusions of Theorem 3.1 still hold for $\ell_{1}(\widehat{F})$ or $\ell_{\infty}(\widehat{F})$ instead of $\ell_{p}(\widehat{F})$ with $q=1$, and on replacing the summations over $k$ by the supremums over $k$ in the case $\ell_{1}(\widehat{F})$. Then, we have the following results:

Theorem 3.2. Let $\tilde{A}=\left(\tilde{a}_{n k}\right)$ be the associated matrix defined by (2.9). Then we have:

(a) if $A \in\left(\ell_{\infty}(\widehat{F}), \ell_{\infty}\right)$, then

$$
0 \leq\left\|L_{A}\right\|_{\chi} \leq \limsup _{n} \sum_{k}\left|\tilde{a}_{n k}\right|
$$

and

$$
L_{A} \text { is compact if } \lim _{n} \sum_{k}\left|\tilde{a}_{n k}\right|=0 \text {; }
$$

(b) if $A \in\left(\ell_{\infty}(\widehat{F}), c_{0}\right)$, then

$$
\left\|L_{A}\right\|_{\chi}=\limsup _{n} \sum_{k}\left|\tilde{a}_{n k}\right|
$$

and

$$
L_{A} \text { is compact if and only if } \lim _{n} \sum_{k}\left|\tilde{a}_{n k}\right|=0 \text {; }
$$

(c) if $A \in\left(\ell_{\infty}(\widehat{F}), c\right)$, then

$$
\frac{1}{2} \cdot \limsup _{n} \sum_{k}\left|\tilde{a}_{n k}-\tilde{a}_{k}\right| \leq\left\|L_{A}\right\|_{\chi} \leq \limsup _{n} \sum_{k}\left|\tilde{a}_{n k}-\tilde{a}_{k}\right|
$$

and

$$
L_{A} \text { is compact if and only if } \lim _{n} \sum_{k}\left|\tilde{a}_{n k}-\tilde{a}_{k}\right|=0 \text {, }
$$

where $\tilde{\alpha}=\left(\tilde{\alpha}_{k}\right)$ with $\tilde{\alpha}_{k}=\lim _{n} \tilde{a}_{n k}$ for all $k \in \mathbb{N}$.

Theorem 3.3. Let $\tilde{A}=\left(\tilde{a}_{n k}\right)$ be the associated matrix defined by (2.9). Then we have 
(a) If $A \in\left(\ell_{1}(\widehat{F}), \ell_{\infty}\right)$, then

and

$$
0 \leq\left\|L_{A}\right\|_{\chi} \leq \limsup _{n}\left(\sup _{k}\left|\tilde{a}_{n k}\right|\right)
$$

$$
L_{A} \text { is compact if } \lim _{n}\left(\sup _{k}\left|\tilde{a}_{n k}\right|\right)=0 .
$$

(b) If $A \in\left(\ell_{1}(\widehat{F}), c_{0}\right)$, then

$$
\left\|L_{A}\right\|_{\chi}=\limsup _{n}\left(\sup _{k}\left|\tilde{a}_{n k}\right|\right)
$$

and

$$
L_{A} \text { is compact if and only if } \lim _{n}\left(\sup _{k}\left|\tilde{a}_{n k}\right|\right)=0 \text {. }
$$

(c) If $A \in\left(\ell_{1}(\widehat{F}), c\right)$, then

$$
\frac{1}{2} \cdot \limsup _{n}\left(\sup _{k}\left|\tilde{a}_{n k}-\tilde{a}_{k}\right|\right) \leq\left\|L_{A}\right\|_{\chi} \leq \limsup _{n}\left(\sup _{k}\left|\tilde{a}_{n k}-\tilde{a}_{k}\right|\right)
$$

and

$$
L_{A} \text { is compact if and only if } \lim _{n}\left(\sup _{k}\left|\tilde{a}_{n k}-\tilde{a}_{k}\right|\right)=0 \text {, }
$$

where $\tilde{\alpha}=\left(\tilde{\alpha}_{k}\right)$ with $\tilde{\alpha}_{k}=\lim _{n} \tilde{a}_{n k}$ for all $k \in \mathbb{N}$.

Morever, as an immediate consequence of Theorem 3.2, we have the following corollary.

Corollary 3.1. If either $A \in\left(\ell_{\infty}(\widehat{F}), c_{0}\right)$ or $A \in\left(\ell_{\infty}(\widehat{F}), c\right)$, then the operator $L_{A}$ is compact.

Proof. Let $A \in\left(\ell_{\infty}(\widehat{F}), c_{0}\right)$. Then, we have by Lemma 2.3 that $\tilde{A} \in\left(\ell_{\infty}, c_{0}\right)$ which implies that $\lim _{n}\left(\sum_{k}\left|\tilde{a}_{n k}\right|\right)=0$, [39]. This leads us with Theorem 3.2(b) to the consequence that $L_{A}$ is compact. Similarly, if $A \in\left(\ell_{\infty}(\widehat{F}), c\right)$ then $\tilde{A} \in\left(\ell_{\infty}, c\right)$ and hence $\lim _{n}\left(\sum_{k}\left|\tilde{a}_{n k}-\tilde{a}_{k}\right|\right)=0$, where $\tilde{\alpha}=\left(\tilde{\alpha}_{k}\right)$ with $\tilde{\alpha}_{k}=\lim _{n} \tilde{a}_{n k}$ for all $k \in \mathbb{N}$. Therefore, we deduce from Theorem 3.2(c) that $L_{A}$ is compact.

Throughout, let $\mathcal{F}_{m}(m \in \mathbb{N})$ be the subcollection of $\mathcal{F}$ consisting of all nonempty and finite subsets of $\mathbb{N}$ with elements that are greater than $m$, that is

$$
\mathcal{F}_{m}=\{n \in N \in \mathcal{F}: n>m \text { for all } n \in \mathbb{N}\} ; \quad m \in \mathbb{N} .
$$

The next lemma [29, Theorem 3.11] gives necessary and sufficient conditions for a matrix transformation from a $B K$ space to $\ell_{1}$ to be compact.

Lemma 3.2. Let $X \supset \varphi$ be a $B K$ space. If $A \in\left(X, \ell_{1}\right)$, then

$$
\lim _{m}\left(\sup _{N \in \mathcal{F}_{m}}\left\|\sum_{n \in N} A_{n}\right\|_{X}^{*}\right) \leq\left\|L_{A}\right\|_{\chi} \leq 4 \cdot \lim _{m}\left(\sup _{N \in \mathcal{F}_{m}}\left\|\sum_{n \in N} A_{n}\right\|_{X}^{*}\right) .
$$


Now, we prove the following result.

Theorem 3.4. Let $1<p<\infty$ and $q=p /(p-1)$. If $A \in\left(\ell_{p}(\widehat{F}), \ell_{1}\right)$, then

$$
\lim _{m}\|A\|_{\left(\ell_{p}(\widehat{F}), \ell_{1}\right)}^{(m)} \leq\left\|L_{A}\right\|_{\chi} \leq 4 \cdot \lim _{m}\|A\|_{\left(\ell_{p}(\widehat{F}), \ell_{1}\right)}^{(m)}
$$

and

$$
L_{A} \text { is compact if and only if } \lim _{m}\|A\|_{\left(\ell_{p}(\widehat{F}), \ell_{1}\right)}^{(m)}=0 \text {, }
$$

where

$$
\|A\|_{\left(\ell_{p}(\widehat{F}), \ell_{1}\right)}^{(m)}=\sup _{N \in \mathcal{F}_{m}}\left(\sum_{k}\left|\sum_{n \in N} \tilde{a}_{n k}\right|^{q}\right)^{1 / q} ; \quad m \in \mathbb{N} .
$$

Proof. It is obvious that (3.11) is obtained by combining Lemmas 2.2(c), 2.3 and 3.2. Also, by using (1.5), we get (3.12) from (3.11).

Theorem 3.5. Let $1 \leq p<\infty$. If $A \in\left(\ell_{1}(\widehat{F}), \ell_{p}\right)$, then

$$
\left\|L_{A}\right\|_{\chi}=\lim _{m}\left(\sup _{k}\left(\sum_{n=m}^{\infty}\left|\tilde{a}_{n k}\right|^{p}\right)^{1 / p}\right)
$$

and

$$
L_{A} \text { is compact if and only if } \lim _{m}\left(\sup _{k}\left(\sum_{n=m}^{\infty}\left|\tilde{a}_{n k}\right|^{p}\right)^{1 / p}\right)=0 \text {, }
$$

Proof. Let us remark that the limit in (3.13) exists by Lemma 2.4.

Now, we write $S=S_{\ell_{1}(\widehat{F})}$. Then, we have by Lemma 1.2 that $L_{A}(S)=A S \in M_{\ell_{p}}$. Thus, it follows from (1.4) and Lemma 1.4 that

$$
\left\|L_{A}\right\|_{\chi}=\chi(A S)=\lim _{m}\left(\sup _{x \in S}\left\|\left(I-P_{m}\right)(A x)\right\|_{\ell_{p}}\right)
$$

where $P_{m}: \ell_{p} \rightarrow \ell_{p}(m \in \mathbb{N})$ is the operator defined by $P_{m}(x)=\left(x_{0}, x_{1}, \ldots, x_{m}, 0,0, \ldots\right)$ for all $x=\left(x_{k}\right) \in \ell_{p}$ and $I$ is the identity operator on $\ell_{p}$.

On the other hand, let $x \in \ell_{1}(\widehat{F})$ be given. Then $y \in \ell_{1}$ and since $A \in\left(\ell_{1}(\widehat{F}), \ell_{p}\right)$, we obtain from Lemma 2.3 that $\tilde{A} \in\left(\ell_{1}, \ell_{p}\right)$ and $A x=\tilde{A} y$. Thus, we have for every 
$m \in \mathbb{N}$ that

$$
\begin{aligned}
\left\|\left(I-P_{m}\right)(A x)\right\|_{\ell_{p}} & =\left\|\left(I-P_{m}\right)(\tilde{A} y)\right\|_{\ell_{p}} \\
& =\left(\sum_{n=m+1}^{\infty}\left|\tilde{A}_{n}(y)\right|^{p}\right)^{1 / p} \\
& =\left(\sum_{n=m+1}^{\infty}\left|\sum_{k} \tilde{a}_{n k} y_{k}\right|^{p}\right)^{1 / p} \\
& \leq \sum_{k}\left(\sum_{n=m+1}^{\infty}\left|\tilde{a}_{n k} y_{k}\right|^{p}\right)^{1 / p} \\
& \leq\|y\|_{\ell_{1}}\left(\sup _{k}\left(\sum_{n=m+1}^{\infty}\left|\tilde{a}_{n k}\right|^{p}\right)^{1 / p}\right) \\
& =\|x\|_{\ell_{1}(\widehat{F})}\left(\sup _{k}\left(\sum_{n=m+1}^{\infty}\left|\tilde{a}_{n k}\right|^{p}\right)^{1 / p}\right) .
\end{aligned}
$$

This yields that

$$
\sup _{x \in S}\left\|\left(I-P_{m}\right)(A x)\right\|_{\ell_{p}} \leq \sup _{k}\left(\sum_{n=m+1}^{\infty}\left|\tilde{a}_{n k}\right|^{p}\right)^{1 / p} ; m \in \mathbb{N} .
$$

Therefore, we deduce from (3.15) that

$$
\left\|L_{A}\right\|_{\chi} \leq \lim _{m}\left(\sup _{k}\left(\sum_{n=m+1}^{\infty}\left|\tilde{a}_{n k}\right|^{p}\right)^{1 / p}\right) \text {. }
$$

To prove the converse inequality, let $c^{(k)} \in \ell_{1}(\widehat{F})$ be such that $\widehat{F} c^{(k)}=e^{(k)}(k \in \mathbb{N})$, that is, $e^{(k)}$ is the $\widehat{F}$-transform of $c^{(k)}$ for each $k \in \mathbb{N}$. Then, we have by Lemma 2.3 that $A c^{(k)}=\tilde{A} e^{(k)}$ for every $k \in \mathbb{N}$.

Now, let $U=\left\{c^{(k)}: k \in \mathbb{N}\right\}$. Then $U \subset S$ and hence $A U \subset A S$ which implies that $\chi(A U) \leq \chi(A S)=\left\|L_{A}\right\|_{\chi}$.

Further, it follows by applying Lemma 1.4 that

$$
\begin{aligned}
\chi(A U) & =\lim _{m}\left(\sup _{k}\left(\left(\sum_{n=m+1}^{\infty}\left|A_{n}\left(c^{(k)}\right)\right|^{p}\right)^{1 / p}\right)\right) \\
& =\lim _{m}\left(\sup _{k}\left(\sum_{n=m+1}^{\infty}\left|\tilde{a}_{n k}\right|^{p}\right)^{1 / p}\right) .
\end{aligned}
$$


Thus, we obtain that

$$
\lim _{m}\left(\sup _{k}\left(\sum_{n=m+1}^{\infty}\left|\tilde{a}_{n k}\right|^{p}\right)^{1 / p}\right) \leq\left\|L_{A}\right\|_{\chi} .
$$

Hence, we get (3.13) by combining (3.16) and (3.17). This completes the proof, since (3.14) is immediate by (1.5) and (3.13).

\section{REFERENCES}

[1] Z. U. Ahmad and M. Mursaleen, Köthe-Toeplitz duals of some new sequence spaces and their matrix maps, Publ. Inst. Math. (Beograd), 42 (56) (1987), 57-61.

[2] B. Altay and F. Başar, The matrix domain and the fine spectrum of the difference operator $\Delta$ on the sequence space $\ell_{p},(0<p<1)$, Commun. Math. Anal. 2(2) (2007), 1-11.

[3] B. Altay, F. Başar and M. Mursaleen, Some generalizations of the space bv $v_{p}$ of $p$-bounded variation sequences, Nonlinear Anal. 68 (2008), 273-28.

[4] F. Başar, Summability Theory and Its Applications, Bentham Science Publishers, e-books, Monographs, İstanbul, 2012.

[5] F. Başar and B. Altay, On the space of sequences of p-bounded variation and related matrix mappings, Ukrainian Math. J. 55 (2003), 136-147.

[6] F. Başar and E. Malkowsky, The characterization of compact operators on spaces of strongly summable and bounded sequences, Appl. Math. Comput. 217 (2011), 5199-5207.

[7] M. Başarır and E. E. Kara, On the B-difference sequence space derived by generalized weighted mean and compact operators, J. Math. Anal. Appl. 391 (2012), 67-81.

[8] M. Başarır and E. E. Kara, On compact operators on the Riesz $B^{(m)}$-difference sequence spaces, Iran. J. Sci. Technol. 35(A4) (2011), 279-285.

[9] M. Başarır and E. E. Kara, On some difference sequence spaces of weighted means and compact operators, Ann. Funct. Anal. 2 (2011), 114-129.

[10] M. Başarır and E. E. Kara, On compact operators on the Riesz $B^{(m)}$-difference sequence spaces II, Iran. J. Sci. Technol. 36(A3) (2012) (Special Issue-Mathematics), 371-376.

[11] B. Choudhary and S. K. Mishra, A note on Köthe-Toeplitz duals of certain sequence spaces and their matrix transformations, Int. J. Math. Math. Sci. 18(4) (1995), 681-688.

[12] R. Çolak, M. Et and E. Malkowsky, Some Topics of Sequence Spaces, Firat University Press, ISBN: 975-394-0386-6, 2004, 1-63.

[13] M. Et, On some difference sequence spaces, Turkish J. Math.7 (1993), 18-24.

[14] A. K. Gaur and M. Mursaleen, Difference sequence spaces, Int. J. Math. Math. Sci. 21(4) (1998), 701-706.

[15] E. E. Kara, Some topological and geometrical properties of new Banach sequence spaces, J. Inequal. Appl. 2013(38) (2013), 15 pages.

[16] E. E. Kara and M. Başarır, On some Euler $B^{(m)}$ difference sequence spaces and compact operators, J. Math. Anal. Appl. 379 (2011), 499-511.

[17] H. Kizmaz, On certain sequence spaces, Canad. Math. Bull. 24(2) (1981), 169-176.

[18] M. Kirişçi and F. Başar, Some new sequence spaces derived by the domain of generalized difference matrix, Comput. Math. Appl. 60 (2010), 1299-1309.

[19] T. Koshy, Fibonacci and Lucas Numbers with Applications, Wiley, 2001.

[20] I. J. Maddox, Continuous and Köthe-Toeplitz duals of certain sequence spaces, Proc. Camb. Phil. Soc. 65 (1965), 431-435.

[21] B. de Malafosse and V. Rakočević, Applications of measure of noncompactness in operators on the spaces $s_{\alpha}, s_{\alpha}^{0}, s_{\alpha}^{c}, \ell_{\alpha}^{p}$, J. Math. Anal. Appl. 323(1) (2006), 131-145. 
[22] E. Malkowsky and V. Rakočević, An introduction into the theory of sequence spaces and measure of noncompactness, Zbornik radova, Matematicki Inst. SANU, Belgrade, 9(17) (2000), 143-234.

[23] E. Malkowsky, Absolute and ordinary Köthe-Toeplitz duals of some sets of sequences and matrix transformations, Publ. Inst. Math. (Beograd), 46(60) (1989), 97-103.

[24] E. Malkowsky and M. Mursaleen, Some matrix transformations between the difference sequence spaces $\Delta c_{0}(p), \Delta c(p)$ and $\Delta \ell_{\infty}(p)$, Filomat, 15 (2001), 353-363.

[25] S. K. Mishra, Matrix maps involving certain sequence spaces, Indian J. Pure Appl. Math. 24(2) (1993), 125-132.

[26] M. Mursaleen and A. K. Noman, Compactness of matrix operators on some new difference sequence spaces, Linear Algebra Appl. 436(1) (2012), 41-52.

[27] M. Mursaleen, Generalized spaces of difference sequences, J. Math. Anal. Appl. 203(3) (1996), 738-745.

[28] M. Mursaleen, A. K. Gaur and A. H. Saifi, Some new sequence spaces and their duals and matrix transformations, Bull. Calcutta Math. Soc. 88(3) (1996), 207-212.

[29] M. Mursaleen and A. K. Noman, Compactness by the Hausdorff measure of noncompactness, Nonlinear Anal. 73(8) (2010), 2541-2557.

[30] M. Mursaleen and A. K. Noman, The Hausdorff measure of noncompactness of matrix operators on some BK spaces, Oper. Matrices, 5(3) (2011), 473-486.

[31] M. Mursaleen, V. Karakaya, H. Polat and N. Şimşek, Measure of noncompactness of matrix operators on some difference sequence spaces of weighted means, Comput. Math. Appl. 62 (2011), 814-820.

[32] M. Mursaleen and S. A. Mohiuddine, Applications of measures of noncompactness to the infinite system of differential equations in $\ell_{p}$ spaces, Nonlinear Anal. 75 (2012), 2111-2115.

[33] M. Mursaleen and A. K. Noman, On $\sigma$-conservative matrices and compact operators on the space $V_{\sigma}$, Appl. Math. Lett. 24 (2011), 1554-1560.

[34] M. Mursaleen and A. K. Noman, Applications of Hausdorff measure of noncompactness in the spaces of generalized means, Math. Inequal. Appl. 16(1) (2013), 207-220.

[35] V. Rakočević, Measures of noncompactness and some applications, Filomat 12(2) (1998), 87-120.

[36] M. A. Sarıöl, On difference sequence spaces, J. Karadeniz Tech. Univ. Fac. Arts Sci. Ser. Math.-Phys. 10 (1987), 63-71.

[37] S. Simons, The sequence spaces $\ell\left(p_{v}\right)$ and $m\left(p_{v}\right)$, Proc. London Math. Soc. 3(15) (1965), 422-436.

[38] A. Sönmez, Some new sequence spaces derived by the domain of the triple band matrix, Comput. Math. Appl. 62(2) (2011), 641-650.

[39] M. Stieglitz and H. Tietz, Matrix transformationen von folgenräumen eine ergebnisübersicht, Math. Z. 154 (1977), 1-16.

${ }^{1}$ Department of Mathematics,

DÜZCE UNIVERSITY,

DÜZCE, TURKEY

E-mail address: eevrenkara@hotmail.com

E-mail address: eevrenkara@duzce.edu.tr

${ }^{2}$ Department of Mathematics,

SAKARYA UNIVERSITY,

SAKARYA, TURKEY

E-mail address: basarir@sakarya.edu.tr

${ }^{3}$ Department of Mathematics,

Aligarh Muslim University,

Aligarh, India

E-mail address: mursaleenm@gmail.com 